\title{
Minimally Supervised Learning of Affective Events Using Discourse Relations
}

\author{
Jun Saito Yugo Murawaki Sadao Kurohashi \\ Graduate School of Informatics, Kyoto University \\ Yoshida-honmachi, Sakyo-ku, Kyoto, 606-8501, Japan \\ \{saito, murawaki, kuro\}@nlp.ist.i.kyoto-u.ac.jp
}

\begin{abstract}
Recognizing affective events that trigger positive or negative sentiment has a wide range of natural language processing applications but remains a challenging problem mainly because the polarity of an event is not necessarily predictable from its constituent words. In this paper, we propose to propagate affective polarity using discourse relations. Our method is simple and only requires a very small seed lexicon and a large raw corpus. Our experiments using Japanese data show that our method learns affective events effectively without manually labeled data. It also improves supervised learning results when labeled data are small.
\end{abstract}

\section{Introduction}

Affective events (Ding and Riloff, 2018) are events that typically affect people in positive or negative ways. For example, getting money and playing sports are usually positive to the experiencers; catching cold and losing one's wallet are negative. Understanding affective events is important to various natural language processing (NLP) applications such as dialogue systems (Shi and $\mathrm{Yu}, 2018$ ), question-answering systems (Oh et al., 2012), and humor recognition (Liu et al., 2018). In this paper, we work on recognizing the polarity of an affective event that is represented by a score ranging from -1 (negative) to 1 (positive).

Learning affective events is challenging because, as the examples above suggest, the polarity of an event is not necessarily predictable from its constituent words. Combined with the unbounded combinatorial nature of language, the non-compositionality of affective polarity entails the need for large amounts of world knowledge, which can hardly be learned from small annotated data.
In this paper, we propose a simple and effective method for learning affective events that only requires a very small seed lexicon and a large raw corpus. As illustrated in Figure 1, our key idea is that we can exploit discourse relations (Prasad et al., 2008) to efficiently propagate polarity from seed predicates that directly report one's emotions (e.g., "to be glad" is positive). Suppose that events $x_{1}$ are $x_{2}$ are in the discourse relation of CAUSE (i.e., $x_{1}$ causes $x_{2}$ ). If the seed lexicon suggests $x_{2}$ is positive, $x_{1}$ is also likely to be positive because it triggers the positive emotion. The fact that $x_{2}$ is known to be negative indicates the negative polarity of $x_{1}$. Similarly, if $x_{1}$ and $x_{2}$ are in the discourse relation of CONCESSION (i.e., $x_{2}$ in spite of $x_{1}$ ), the reverse of $x_{2}$ 's polarity can be propagated to $x_{1}$. Even if $x_{2}$ 's polarity is not known in advance, we can exploit the tendency of $x_{1}$ and $x_{2}$ to be of the same polarity (for CAUSE) or of the reverse polarity (for CONCESSION) although the heuristic is not exempt from counterexamples. We transform this idea into objective functions and train neural network models that predict the polarity of a given event.

We trained the models using a Japanese web corpus. Given the minimum amount of supervision, they performed well. In addition, the combination of annotated and unannotated data yielded a gain over a purely supervised baseline when labeled data were small.

\section{Related Work}

Learning affective events is closely related to sentiment analysis. Whereas sentiment analysis usually focuses on the polarity of what are described (e.g., movies), we work on how people are typically affected by events. In sentiment analysis, much attention has been paid to compositionality. Word-level polarity (Takamura et al., 2005; 


\begin{tabular}{|c|c|c|c|}
\hline Type & Former event & Latter event & Relation \\
\hline \multirow{2}{*}{$\mathrm{AL}$} & 試合に勝つ ([I] win the game) & 嬉しい ([I] am glad) & \multirow[b]{2}{*}{ CAUSE } \\
\hline & $+1 \leftarrow$ Propaga & $\begin{array}{l}\text { e same polarity } \\
+1\end{array}$ & \\
\hline \multirow{2}{*}{$\mathrm{AL}$} & ピクニックに行く $([\mathrm{I}]$ go to a picnic $)$ & 天気が心配だ ([I] am worried about the weather) & \multirow{2}{*}{ CONCESSION } \\
\hline & Propagate & reverse polarity & \\
\hline \multirow{2}{*}{ CA } & 暖房がない (There is no heating) & 寒い $([\mathrm{I}] \underset{\uparrow}{\mathrm{A}}$ cold $)$ & \multirow{2}{*}{ CAUSE } \\
\hline & Encourage then & have the same polarity & \\
\hline \multirow[t]{2}{*}{$\mathrm{CO}$} & 視力が良い ([I] have good eyes) & よく見えない ([I] cannot see [it] well) & \multirow[t]{2}{*}{ CONCESSION } \\
\hline & Encourage them & ive the reverse polarity & \\
\hline
\end{tabular}

Figure 1: An overview of our method. We focus on pairs of events, the former events and the latter events, which are connected with a discourse relation, CAUSE or CONCESSION. Dropped pronouns are indicated by brackets in English translations. We divide the event pairs into three types: AL, CA, and CO. In AL, the polarity of a latter event is automatically identified as either positive or negative, according to the seed lexicon (the positive word is colored red and the negative word blue). We propagate the latter event's polarity to the former event. The same polarity as the latter event is used for the discourse relation CAUSE, and the reversed polarity for CONCESSION. In $\mathrm{CA}$ and $\mathrm{CO}$, the latter event's polarity is not known. Depending on the discourse relation, we encourage the two events' polarities to be the same (CA) or reversed (CO). Details are given in Section 3.2.

Wilson et al., 2005; Baccianella et al., 2010) and the roles of negation and intensification (Reitan et al., 2015; Wilson et al., 2005; Zhu et al., 2014) are among the most important topics. In contrast, we are more interested in recognizing the sentiment polarity of an event that pertains to commonsense knowledge (e.g., getting money and catching cold).

Label propagation from seed instances is a common approach to inducing sentiment polarities. While Takamura et al. (2005) and Turney (2002) worked on word- and phrase-level polarities, Ding and Riloff (2018) dealt with event-level polarities. Takamura et al. (2005) and Turney (2002) linked instances using co-occurrence information and/or phrase-level coordinations (e.g., " $A$ and $B$ " and " $A$ but $B$ "). We shift our scope to event pairs that are more complex than phrase pairs, and consequently exploit discourse connectives as eventlevel counterparts of phrase-level conjunctions.

Ding and Riloff (2018) constructed a network of events using word embedding-derived similarities. Compared with this method, our discourse relation-based linking of events is much simpler and more intuitive.

Some previous studies made use of document structure to understand the sentiment. Shimizu et al. (2018) proposed a sentiment-specific pretraining strategy using unlabeled dialog data (tweet-reply pairs). Kaji and Kitsuregawa (2006) proposed a method of building a polarity-tagged corpus (ACP Corpus). They automatically gath- ered sentences that had positive or negative opinions utilizing HTML layout structures in addition to linguistic patterns. Our method depends only on raw texts and thus has wider applicability.

\section{Proposed Method}

\subsection{Polarity Function}

Our goal is to learn the polarity function $p(x)$, which predicts the sentiment polarity score of an event $x$. We approximate $p(x)$ by a neural network with the following form:

$$
p(x)=\tanh (\operatorname{Linear}(\operatorname{Encoder}(x))) .
$$

Encoder outputs a vector representation of the event $x$. Linear is a fully-connected layer and transforms the representation into a scalar. tanh is the hyperbolic tangent and transforms the scalar into a score ranging from -1 to 1 . In Section 4.2, we consider two specific implementations of Encoder.

\subsection{Discourse Relation-Based Event Pairs}

Our method requires a very small seed lexicon and a large raw corpus. We assume that we can automatically extract discourse-tagged event pairs, $\left(x_{i 1}, x_{i 2}\right)(i=1, \cdots)$ from the raw corpus. We refer to $x_{i 1}$ and $x_{i 2}$ as former and latter events, respectively. As shown in Figure 1, we limit our scope to two discourse relations: CAUSE and CONCESSION.

The seed lexicon consists of positive and negative predicates. If the predicate of an extracted 
event is in the seed lexicon and does not involve complex phenomena like negation, we assign the corresponding polarity score $(+1$ for positive events and -1 for negative events) to the event. We expect the model to automatically learn complex phenomena through label propagation. Based on the availability of scores and the types of discourse relations, we classify the extracted event pairs into the following three types.

AL (Automatically Labeled Pairs) The seed lexicon matches (1) the latter event but (2) not the former event, and (3) their discourse relation type is CAUSE or CONCESSION. If the discourse relation type is CAUSE, the former event is given the same score as the latter. Likewise, if the discourse relation type is CONCESSION, the former event is given the opposite of the latter's score. They are used as reference scores during training.

CA (CAuse Pairs) The seed lexicon matches neither the former nor the latter event, and their discourse relation type is CAUSE. We assume the two events have the same polarities.

CO (Concession Pairs) The seed lexicon matches neither the former nor the latter event, and their discourse relation type is CONCESSION. We assume the two events have the reversed polarities.

\subsection{Loss Functions}

Using AL, CA, and CO data, we optimize the parameters of the polarity function $p(x)$. We define a loss function for each of the three types of event pairs and sum up the multiple loss functions.

We use mean squared error to construct loss functions. For the AL data, the loss function is defined as:

$$
\begin{aligned}
\mathcal{L}_{\mathrm{AL}}= & \frac{1}{N_{\mathrm{AL}}} \sum_{i=1}^{N_{\mathrm{AL}}}\left(r_{i 2}-p\left(x_{i 2}\right)\right)^{2} \\
& +\lambda_{\mathrm{AL}} \frac{1}{N_{\mathrm{AL}}} \sum_{i=1}^{N_{\mathrm{AL}}}\left(r_{i 1}-p\left(x_{i 1}\right)\right)^{2},
\end{aligned}
$$

where $x_{i 1}$ and $x_{i 2}$ are the $i$-th pair of the AL data. $r_{i 1}$ and $r_{i 2}$ are the automatically-assigned scores of $x_{i 1}$ and $x_{i 2}$, respectively. $N_{\mathrm{AL}}$ is the total number of AL pairs, and $\lambda_{\mathrm{AL}}$ is a hyperparameter.
For the CA data, the loss function is defined as:

$$
\begin{aligned}
\mathcal{L}_{\mathrm{CA}}= & \lambda_{\mathrm{CA}} \frac{1}{N_{\mathrm{CA}}} \sum_{i=1}^{N_{\mathrm{CA}}}\left(p\left(y_{i 1}\right)-p\left(y_{i 2}\right)\right)^{2} \\
& +\mu \frac{1}{N_{\mathrm{CA}}} \sum_{i=1}^{N_{\mathrm{CA}}} \sum_{u \in\left\{y_{i 1}, y_{i 2}\right\}}\left(1-p(u)^{2}\right) .
\end{aligned}
$$

$y_{i 1}$ and $y_{i 2}$ are the $i$-th pair of the CA pairs. $N_{\mathrm{CA}}$ is the total number of CA pairs. $\lambda_{\mathrm{CA}}$ and $\mu$ are hyperparameters. The first term makes the scores of the two events closer while the second term prevents the scores from shrinking to zero.

The loss function for the CO data is defined analogously:

$$
\begin{aligned}
\mathcal{L}_{\mathrm{CO}}= & \lambda_{\mathrm{CO}} \frac{1}{N_{\mathrm{CO}}} \sum_{i=1}^{N_{\mathrm{CO}}}\left(p\left(z_{i 1}\right)+p\left(z_{i 2}\right)\right)^{2} \\
& +\mu \frac{1}{N_{\mathrm{CO}}} \sum_{i=1}^{N_{\mathrm{CO}}} \sum_{u \in\left\{z_{i 1}, z_{i 2}\right\}}\left(1-p(u)^{2}\right) .
\end{aligned}
$$

The difference is that the first term makes the scores of the two events distant from each other.

\section{Experiments}

\subsection{Dataset}

\subsubsection{AL, CA, and CO}

As a raw corpus, we used a Japanese web corpus that was compiled through the procedures proposed by Kawahara and Kurohashi (2006). To extract event pairs tagged with discourse relations, we used the Japanese dependency parser $\mathrm{KNP}^{1}$ and in-house postprocessing scripts (Saito et al., 2018). KNP used hand-written rules to segment each sentence into what we conventionally called clauses (mostly consecutive text chunks), each of which contained one main predicate. KNP also identified the discourse relations of event pairs if explicit discourse connectives (Prasad et al., 2008) such as “ので” (because) and “のに” (in spite of) were present. We treated Cause/Reason (原 因 - 理由) and Condition (条件) in the original tagset (Kawahara et al., 2014) as CAUSE and Concession (逆接) $)^{2}$ as CONCESSION, respectively. Here is an example of event pair extraction.

\footnotetext{
${ }^{1}$ http://nlp.ist.i.kyoto-u.ac.jp/EN/ index.php?KNP

${ }^{2}$ To be precise, this discourse type is semantically broader than Concession and extends to the area of Contrast.
} 


\begin{tabular}{lc}
\hline \multicolumn{1}{c}{ Type of pairs } & \# of pairs \\
\hline AL (Automatically Labeled Pairs) & $1,000,000$ \\
CA (CAUSE Pairs) & $5,000,000$ \\
CO (CONCESSION Pairs) & $5,000,000$ \\
\hline
\end{tabular}

Table 1: Statistics of the AL, CA, and CO datasets.

（1）重大な失敗を犯したので、仕事をクビ になった。

Because [I] made a serious mistake, [I] got fired.

From this sentence, we extracted the event pair of “重大な失敗を犯す” ([I] make a serious mistake) and “仕事をクビになる” ([I] get fired), and tagged it with CAUSE.

We constructed our seed lexicon consisting of 15 positive words and 15 negative words, as shown in Section A.1. From the corpus of about 100 million sentences, we obtained 1.4 millions event pairs for AL, 41 millions for CA, and 6 millions for $\mathrm{CO}$. We randomly selected subsets of AL event pairs such that positive and negative latter events were equal in size. We also sampled event pairs for each of CA and CO such that it was five times larger than AL. The results are shown in Table 1.

\subsubsection{ACP (ACP Corpus)}

We used the latest version ${ }^{3}$ of the ACP Corpus (Kaji and Kitsuregawa, 2006) for evaluation. It was used for (semi-)supervised training as well. Extracted from Japanese websites using HTML layouts and linguistic patterns, the dataset covered various genres. For example, the following two sentences were labeled positive and negative, respectively:

(2) 作業が楽だ。

The work is easy.

(3) 駐車場がない。

There is no parking lot.

Although the ACP corpus was originally constructed in the context of sentiment analysis, we found that it could roughly be regarded as a collection of affective events. We parsed each sentence and extracted the last clause in it. The train/dev/test split of the data is shown in Table 2. The objective function for supervised training is:

$$
\mathcal{L}_{\mathrm{ACP}}=\frac{1}{N_{\mathrm{ACP}}} \sum_{i=1}^{N_{\mathrm{ACP}}}\left(R_{i}-p\left(v_{i}\right)\right)^{2},
$$

\footnotetext{
${ }^{3}$ The dataset was obtained from Nobuhiro Kaji via personal communication.
}

\begin{tabular}{ccr}
\hline Dataset & Event polarity & \# of events \\
\hline \multirow{2}{*}{ Train } & Positive & 299,834 \\
& Negative & 300,164 \\
\hline \multirow{2}{*}{ Dev } & Positive & 50,118 \\
& Negative & 49,882 \\
\hline \multirow{2}{*}{ Test } & Positive & 50,046 \\
& Negative & 49,954 \\
\hline
\end{tabular}

Table 2: Details of the ACP dataset.

where $v_{i}$ is the $i$-th event, $R_{i}$ is the reference score of $v_{i}$, and $N_{\mathrm{ACP}}$ is the number of the events of the ACP Corpus.

To optimize the hyperparameters, we used the dev set of the ACP Corpus. For the evaluation, we used the test set of the ACP Corpus. The model output was classified as positive if $p(x)>0$ and negative if $p(x) \leq 0$.

\subsection{Model Configurations}

As for Encoder, we compared two types of neural networks: BiGRU and BERT. GRU (Cho et al., 2014) is a recurrent neural network sequence encoder. BiGRU reads an input sequence forward and backward and the output is the concatenation of the final forward and backward hidden states.

BERT (Devlin et al., 2019) is a pre-trained multi-layer bidirectional Transformer (Vaswani et al., 2017) encoder. Its output is the final hidden state corresponding to the special classification tag ( [CLS ] ). For the details of Encoder, see Sections A.2.

We trained the model with the following four combinations of the datasets: $\mathrm{AL}, \mathrm{AL}+\mathrm{CA}+\mathrm{CO}$ (two proposed models), ACP (supervised), and $\mathrm{ACP}+\mathrm{AL}+\mathrm{CA}+\mathrm{CO}$ (semi-supervised). The corresponding objective functions were: $\mathcal{L}_{\mathrm{AL}}, \mathcal{L}_{\mathrm{AL}}+$ $\mathcal{L}_{\mathrm{CA}}+\mathcal{L}_{\mathrm{CO}}, \mathcal{L}_{\mathrm{ACP}}$, and $\mathcal{L}_{\mathrm{ACP}}+\mathcal{L}_{\mathrm{AL}}+\mathcal{L}_{\mathrm{CA}}+$ $\mathcal{L}_{\mathrm{CO}}$.

\subsection{Results and Discussion}

Table 3 shows accuracy. As the Random baseline suggests, positive and negative labels were distributed evenly. The Random+Seed baseline made use of the seed lexicon and output the corresponding label (or the reverse of it for negation) if the event's predicate is in the seed lexicon. We can see that the seed lexicon itself had practically no impact on prediction.

The models in the top block performed considerably better than the random baselines. The performance gaps with their (semi-)supervised counterparts, shown in the middle block, were less than 


\begin{tabular}{llc}
\hline Training dataset & Encoder & Acc \\
\hline \multirow{2}{*}{$\mathrm{AL}$} & BiGRU & 0.843 \\
& BERT & 0.863 \\
$\mathrm{AL}+\mathrm{CA}+\mathrm{CO}$ & BiGRU & $\mathbf{0 . 8 6 6}$ \\
& BERT & 0.835 \\
\hline \multirow{2}{*}{$\mathrm{ACP}$} & BiGRU & 0.919 \\
& BERT & $\mathbf{0 . 9 3 3}$ \\
$\mathrm{ACP}+\mathrm{AL}+\mathrm{CA}+\mathrm{CO}$ & BiGRU & 0.917 \\
& BERT & 0.913 \\
\hline \hline Random & & 0.500 \\
Random+Seed & & 0.503 \\
\hline
\end{tabular}

Table 3: Performance of various models on the ACP test set.

\begin{tabular}{llc}
\hline Training dataset & Encoder & Acc \\
\hline ACP $(6 \mathrm{~K})$ & \multirow{2}{*}{ BERT } & 0.876 \\
+ $\mathrm{AL}$ & & $\mathbf{0 . 8 8 6}$ \\
\hline $\mathrm{ACP}(6 \mathrm{~K})$ & \multirow{2}{*}{$\mathrm{BiGRU}$} & 0.830 \\
+ $\mathrm{AL}+\mathrm{CA}+\mathrm{CO}$ & & $\mathbf{0 . 8 7 9}$ \\
\hline
\end{tabular}

Table 4: Results for small labeled training data. Given the performance with the full dataset, we show BERT trained only with the AL data.

7\%. This demonstrates the effectiveness of discourse relation-based label propagation.

Comparing the model variants, we obtained the highest score with the BiGRU encoder trained with the $\mathrm{AL}+\mathrm{CA}+\mathrm{CO}$ dataset. BERT was competitive but its performance went down if $\mathrm{CA}$ and $\mathrm{CO}$ were used in addition to AL. We conjecture that BERT was more sensitive to noises found more frequently in $\mathrm{CA}$ and $\mathrm{CO}$.

Contrary to our expectations, supervised models (ACP) outperformed semi-supervised models $(\mathrm{ACP}+\mathrm{AL}+\mathrm{CA}+\mathrm{CO})$. This suggests that the training set of 0.6 million events is sufficiently large for training the models. For comparison, we trained the models with a subset $(6,000$ events) of the ACP dataset. As the results shown in Table 4 demonstrate, our method is effective when labeled data are small.

The result of hyperparameter optimization for the BiGRU encoder was as follows:

$$
\lambda_{\mathrm{AL}}=1, \lambda_{\mathrm{CA}}=0.35, \lambda_{\mathrm{CO}}=1, \mu=0.5 .
$$

As the CA and $\mathrm{CO}$ pairs were equal in size (Table $1), \lambda_{\mathrm{CA}}$ and $\lambda_{\mathrm{CO}}$ were comparable values. $\lambda_{\mathrm{CA}}$ was about one-third of $\lambda_{\mathrm{CO}}$, and this indicated that the CA pairs were noisier than the $\mathrm{CO}$ pairs. A major type of CA pairs that violates our assumption was in the form of " problem $_{\text {negative }}$ causes

\begin{tabular}{lr}
\hline \multicolumn{1}{c}{ Input event } & Polarity \\
\hline 道に迷う ([I] get lost) & -0.771 \\
道に迷わない ([I] don't get lost) & 0.835 \\
\hline 笑う ([I] laugh) & 0.624 \\
笑われる ([I] am laughed at) & -0.687 \\
\hline 脂肪を落とす ([I] lose body fat) & 0.452 \\
肩を落とす ([I] feel disappointed) & -0.653 \\
\hline
\end{tabular}

Table 5: Examples of polarity scores predicted by the BiGRU model trained with AL+CA+CO.

solution $_{\text {positive": }}$

（4） (悪いところがある，よくなるように努力 する)

(there is a bad point, [I] try to improve [it])

The polarities of the two events were reversed in spite of the CAUSE relation, and this lowered the value of $\lambda_{\mathrm{CA}}$.

Some examples of model outputs are shown in Table 5. The first two examples suggest that our model successfully learned negation without explicit supervision. Similarly, the next two examples differ only in voice but the model correctly recognized that they had opposite polarities. The last two examples share the predicate “落とす”, (drop) and only the objects are different. The second event “肩を落とす” (lit. drop one's shoulders) is an idiom that expresses a disappointed feeling. The examples demonstrate that our model correctly learned non-compositional expressions.

\section{Conclusion}

In this paper, we proposed to use discourse relations to effectively propagate polarities of affective events from seeds. Experiments show that, even with a minimal amount of supervision, the proposed method performed well.

Although event pairs linked by discourse analysis are shown to be useful, they nevertheless contain noises. Adding linguistically-motivated filtering rules would help improve the performance.

\section{Acknowledgments}

We thank Nobuhiro Kaji for providing the ACP Corpus and Hirokazu Kiyomaru and Yudai Kishimoto for their help in extracting event pairs. This work was partially supported by Yahoo! Japan Corporation. 


\section{References}

Stefano Baccianella, Andrea Esuli, and Fabrizio Sebastiani. 2010. Sentiwordnet 3.0: An enhanced lexical resource for sentiment analysis and opinion mining. In Proceedings of the seventh edition of the Language Resources and Evaluation Conference.

Kyunghyun Cho, Bart van Merrienboer, Caglar Gulcehre, Dzmitry Bahdanau, Fethi Bougares, Holger Schwenk, and Yoshua Bengio. 2014. Learning phrase representations using RNN encoder-decoder for statistical machine translation. In Proceedings of the 2014 Conference on Empirical Methods in Natural Language Processing (EMNLP), pages 17241734, Doha, Qatar. Association for Computational Linguistics.

Jacob Devlin, Ming-Wei Chang, Kenton Lee, and Kristina Toutanova. 2019. BERT: Pre-training of deep bidirectional transformers for language understanding. In Proceedings of the 2019 Conference of the North American Chapter of the Association for Computational Linguistics: Human Language Technologies, Volume 1 (Long and Short Papers), pages 4171-4186, Minneapolis, Minnesota. Association for Computational Linguistics.

Haibo Ding and Ellen Riloff. 2018. Weakly supervised induction of affective events by optimizing semantic consistency. In Proceedings of the 32nd AAAI Conference on Artificial Intelligence.

Nobuhiro Kaji and Masaru Kitsuregawa. 2006. Automatic construction of polarity-tagged corpus from html documents. In Proceedings of the COLING/ACL on Main Conference Poster Sessions, COLING-ACL '06, pages 452-459, Stroudsburg, PA, USA. Association for Computational Linguistics.

Daisuke Kawahara and Sadao Kurohashi. 2006. Case frame compilation from the web using highperformance computing. In Proceedings of the Fifth International Conference on Language Resources and Evaluation (LREC'06), Genoa, Italy. European Language Resources Association (ELRA).

Daisuke Kawahara, Yuichiro Machida, Tomohide Shibata, Sadao Kurohashi, Hayato Kobayashi, and Manabu Sassano. 2014. Rapid development of a corpus with discourse annotations using two-stage crowdsourcing. In Proceedings of COLING 2014, the 25th International Conference on Computational Linguistics: Technical Papers, pages 269278, Dublin, Ireland. Dublin City University and Association for Computational Linguistics.

Diederik Kingma and Jimmy Ba. 2014. Adam: A method for stochastic optimization. International Conference on Learning Representations.

Lizhen Liu, Donghai Zhang, and Wei Song. 2018. Modeling sentiment association in discourse for humor recognition. In Proceedings of the 56th Annual Meeting of the Association for Computational
Linguistics (Volume 2: Short Papers), pages 586591, Melbourne, Australia. Association for Computational Linguistics.

Jong-Hoon Oh, Kentaro Torisawa, Chikara Hashimoto, Takuya Kawada, Stijn De Saeger, Jun'ichi Kazama, and Yiou Wang. 2012. Why question answering using sentiment analysis and word classes. In Proceedings of the 2012 Conference on Empirical Methods on Natural Language Processing and Computational Natural Language Learning.

Rashmi Prasad, Nikhil Dinesh, Alan Lee, Eleni Miltsakaki, Livio Robaldo, Aravind Joshi, and Bonnie Webber. 2008. The Penn discourse TreeBank 2.0. In Proceedings of the 2012 Conference on Empirical Methods on Natural Language Processing and Computational Natural Language Learning.

Johan Reitan, Jørgen Faret, Björn Gambäck, and Lars Bungum. 2015. Negation scope detection for twitter sentiment analysis. In Proceedings of the 9th Workshop on Computational Approaches to Subjectivity, Sentiment and Social Media Analysis.

Jun Saito, Tomohiro Sakaguchi, Tomohide Shibata, Daisuke Kawahara, and Sadao Kurohashi. 2018. Design and visualization of linguistic information unit based on predicate-argument structure. In Proceedings of the 24th Annual Meeting of Natural Language Processing (in Japanese).

Rico Sennrich, Barry Haddow, and Alexandra Birch. 2016. Neural machine translation of rare words with subword units. In Proceedings of the 54th Annual Meeting of the Association for Computational Linguistics (Volume 1: Long Papers), pages 17151725, Berlin, Germany. Association for Computational Linguistics.

Weiyan Shi and Zhou Yu. 2018. Sentiment adaptive end-to-end dialog systems. In Proceedings of the 56th Annual Meeting of the Association for Computational Linguistics (Volume 1: Long Papers), pages 1509-1519, Melbourne, Australia. Association for Computational Linguistics.

Toru Shimizu, Nobuyuki Shimizu, and Hayato Kobayashi. 2018. Pretraining sentiment classifiers with unlabeled dialog data. In Proceedings of the 56th Annual Meeting of the Association for Computational Linguistics (Volume 2: Short Papers), pages 764-770, Melbourne, Australia. Association for Computational Linguistics.

Ilya Sutskever, James Martens, George Dahl, and Geoffrey Hinton. 2013. On the importance of initialization and momentum in deep learning. In Proceedings of the 30th International Conference on Machine Learning, volume 28 of Proceedings of Machine Learning Research, pages 1139-1147, Atlanta, Georgia, USA. PMLR.

Hiroya Takamura, Takashi Inui, and Manabu Okumura. 2005. Extracting semantic orientations of words us- 
ing spin model. In Proceedings of the 43rd Annual Meeting on Association for Computational Linguistics, ACL '05, pages 133-140, Stroudsburg, PA, USA. Association for Computational Linguistics.

Peter D. Turney. 2002. Thumbs up or thumbs down?: Semantic orientation applied to unsupervised classification of reviews. In Proceedings of the 40th Annual Meeting on Association for Computational Linguistics, ACL '02, pages 417-424, Stroudsburg, PA, USA. Association for Computational Linguistics.

Ashish Vaswani, Noam Shazeer, Niki Parmar, Jakob Uszkoreit, Llion Jones, Aidan N Gomez, Ł ukasz Kaiser, and Illia Polosukhin. 2017. Attention is all you need. In I. Guyon, U. V. Luxburg, S. Bengio, H. Wallach, R. Fergus, S. Vishwanathan, and R. Garnett, editors, Advances in Neural Information Processing Systems 30, pages 5998-6008. Curran Associates, Inc.

Theresa Wilson, Janyce Wiebe, and Paul Hoffmann. 2005. Recognizing contextual polarity in phraselevel sentiment analysis. In Proceedings of the Conference on Human Language Technology and Empirical Methods in Natural Language Processing, HLT '05, pages 347-354, Stroudsburg, PA, USA. Association for Computational Linguistics.

Xiaodan Zhu, Hongyu Guo, Saif Mohammad, and Svetlana Kiritchenko. 2014. An empirical study on the effect of negation words on sentiment. In Proceedings of the 52nd Annual Meeting of the Association for Computational Linguistics (Volume 1: Long Papers), pages 304-313, Baltimore, Maryland. Association for Computational Linguistics. 


\section{A Appendices}

\section{A.1 Seed Lexicon}

Positive Words 喜ぶ (rejoice), 嬉しい (be glad), 楽しい (be pleasant), 幸せ (be happy), 感 動 (be impressed), 興奮 (be excited), 懐かしい (feel nostalgic), 好き (like), 尊敬 (respect), 安心 (be relieved), 感心 (admire), 落ち着く (be calm), 満足 (be satisfied), 癒される (be healed), and スッキリ (be refreshed).

Negative Words 怒る (get angry), 悲しい (be sad), 寂しい (be lonely), 怖い (be scared), 不安 (feel anxious), 恥ずかしい (be embarrassed), 嫌 (hate), 落ち込む (feel down), 退屈 (be bored), 絶望 (feel hopeless), 辛い (have a hard time), 困 る (have trouble), 憂鬱 (be depressed), 心配 (be worried), and 情けない (be sorry).

\section{A.2 Settings of Encoder}

BiGRU The dimension of the embedding layer was 256 . The embedding layer was initialized with the word embeddings pretrained using the Web corpus. The input sentences were segmented into words by the morphological analyzer Juman++. ${ }^{4}$ The vocabulary size was 100,000 . The number of hidden layers was 2 . The dimension of hidden units was 256. The optimizer was Momentum SGD (Sutskever et al., 2013). The mini-batch size was 1024 . We ran 100 epochs and selected the snapshot that achieved the highest score for the dev set.

BERT We used a Japanese BERT model ${ }^{5}$ pretrained with Japanese Wikipedia. The input sentences were segmented into words by Juman++, and words were broken into subwords by applying BPE (Sennrich et al., 2016). The vocabulary size was 32,000 . The maximum length of an input sequence was 128 . The number of hidden layers was 12 . The dimension of hidden units was 768 . The number of self-attention heads was 12 . The optimizer was Adam (Kingma and Ba, 2014). The mini-batch size was 32 . We ran 1 epoch.

\footnotetext{
${ }^{4}$ http://nlp.ist.i.kyoto-u.ac.jp/EN/ index.php? JUMAN++

${ }^{5}$ http://nlp.ist.i.kyoto-u.ac.jp/ index. php?BERTE $6 \% 97 \% \mathrm{~A} 5 \mathrm{E} 6 \% 9 \mathrm{C} \% \mathrm{AC} \div \mathrm{E} 8 \% \mathrm{AA} \div$ $9 \mathrm{EPretrained} \mathrm{E} 3 \% 83 \div \mathrm{A} 2 \% \mathrm{E} 3 \% 83 \div 87 \% \mathrm{E} 3 \div 83 \div \mathrm{AB}$ (in Japanese)
} 